\title{
Efeitos da utilização de prebióticos (oligossacarídeos) na saúde da criança
}

\author{
Effects of prebiotics (oligosaccharides) use on child's health
}

Elisabete Millani', Tulio Konstantyner ${ }^{2}$, José Augusto de A. C. Taddei ${ }^{3}$

\section{RESUMO}

Objetivo: Avaliar os efeitos da utilização de prebióticos (oligossacarídeos) na saúde da criança, suas aplicações e os possíveis mecanismos envolvidos.

Fontes de dados: Os artigos para esta revisão foram selecionados das bases de dados virtuais de saúde da Biblioteca Regional de Medicina (Bireme), que incluem Lilacs, Medline e SciELO. Foram pesquisados artigos entre 1998 e 2008, compreendendo a faixa etária até 12 anos.

Síntese dos dados: Os oligossacarídeos prebióticos em produtos alimentares demonstraram efeitos benéficos quanto ao aumento do número total de bifidobactérias, redução do número de microorganismos patogênicos no intestino e melhora da consistência das fezes e frequência das evacuações. Tais efeitos sugerem a recomendação de seu uso em crianças constipadas. Foram verificados resultados benéficos em diversos estudos quanto ao menor desenvolvimento de alergias, infecções respiratórias, febre, irritabilidade, flatulência, diarreia, regurgitação, vômito, cólica e choro, menor uso de antibióticos e melhora nas características de fermentação de ácidos graxos e pH fecal. Entretanto, o número de artigos encontrados aponta para a necessidade de realizar mais investigações. Estudos sobre absorção de cálcio foram contraditórios, não permitindo conclusões definitivas. Quanto à tolerância, foi evidenciado o uso de quantidades diversas de suplemento em crianças de diferentes faixas etárias e características clínicas, impossibilitando identificar a segurança desses produtos.

Conclusões: Apesar dos prebióticos parecerem promissores e oferecerem efeitos benéficos em determinadas situações, alguns aspectos duvidosos devem ser investigados de forma a avaliar o real efeito e segurança de sua utilização em curto e longo prazo, a fim de comprovar a ausência de consequências prejudiciais à saúde da criança.
Palavras-chave: oligossacarídeos; saúde da criança; suplementos dietéticos; segurança alimentar e nutricional.

\section{ABSTRACT}

Objective: To evaluate the use of prebiotics (oligosaccharides) in child health, their applications and possible mechanisms of action.

Data source: The articles for this review were electronically selected from the Biblioteca Regional de Medicina (Bireme) virtual library database, which includes Lilacs, Medline and SciELO databases. Search included articles on the subject from 1998 to 2008 comprising children up to 12 years old.

Data synthesis: The prebiotic oligosaccharides in food products provided beneficial effects that included increase in total number of bifidobacteria, reduction in the number of pathogenic intestinal microorganisms; improvement of excrement consistency and in the frequency of evacuations These effects suggest their recommendation for constipated children. Beneficial results were verified in several studies concerning the reduction of allergies development, respiratory infections, fever, irritability, flatulence, diarrhea, regurgitation, vomits, colic, cry and use of antibiotics, with improvement of fermentation characteristics of fatty acids and fecal pH. Nevertheless, the number of studies found indicates the need for further investigation. Studies on calcium absorption were contradictory. Concerning tolerance, it is impossible to verify the safety of these products due to the use of diverse quantities of supplement for children from different age ranges and clinical characteristics

Conclusions: Although prebiotics seem promising and provide beneficial effects in certain situations, they deserve further investigation to evaluate their actual ef-
Instituição: Departamento Pediatria da Escola Paulista de Medicina da Universidade Federal de São Paulo (Unifesp-EPM), São Paulo, SP, Brasil

${ }^{1}$ Nutricionista; Especialista em Saúde, Nutrição e Alimentação na Infância e Adolescência pela Unifesp-EPM, São Paulo, SP, Brasil

2Pediatra; Mestre em Ciências e Doutorando da Unifesp-EPM, São Paulo, SP, Brasil

${ }^{3}$ Professor associado da Disciplina de Nutrologia do Departamento de Pediatria da Unifesp-EPM, São Paulo, SP, Brasil
Endereço para correspondência:

José Augusto de A. C. Taddei

Endereço: Rua Loefgreen 1647 - Vila Clementino

CEP 04040-032 - São Paulo/SP

E-mail: taddei.dped@epm.br

Recebido em: 23/9/08

Aprovado em: 19/12/08 
ficacy and safety on immediate and long term conditions, in order to confirm the absence of harmful consequences for children's health.

Key-words: oligosaccharides; child health (public health); dietary supplements; food security.

\section{Introdução}

O consumo do leite materno está associado à baixa incidência de doenças infecciosas e alérgicas em recém-nascidos $(\mathrm{RN})$ e lactentes. A flora intestinal de crianças amamentadas apresenta propriedades anti-infecciosas e, consequentemente, constitui fator importante de estimulação para o desenvolvimento pós-natal do sistema imune. O efeito do leite materno na flora intestinal não pode ser atribuído a um único componente ${ }^{(1)}$.

Os oligossacarídeos são o terceiro maior componente contínuo e abundante presente no leite materno, após a lactose e os lipídeos ${ }^{(2)}$, e seu índice no leite materno varia conforme a composição genética da mãe, a duração e o período da lactação(3). Estudos comparativos das fezes de crianças em aleitamento materno e crianças alimentadas com fórmulas infantis demonstram que crianças amamentadas, ao contrário das demais, apresentam flora intestinal com alta prevalência de bifidobactérias e lactobacilos ${ }^{(4)}$. Além disso, há evidência de que os oligossacarídeos humanos inibem a adesão e a ação dos patógenos na superfície epitelial, tornando-os importantes componentes de proteção à saúde infantil ${ }^{(1)}$.

O leite materno acarreta o crescimento da flora intestinal 'normal', como bifidobactérias e lactobacilos, por meio da atuação do que se convencionou chamar 'bifidus factor'. Essa atividade se faz em função de oligossacarídeos como a glicoproteína que contém glicose, galactose, frutose e Nacetilgalactosamina, que propicia o crescimento de bífidobactérias e lactobacilos. $\mathrm{O}$ aumento da concentração desses microorganismos no trato gastrintestinal é considerado benéfico, acarretando a síntese de moléculas que apresentam efeito antibacteriano e antibiótico, a inibição de crescimento de bactérias patogênicas, a resistência a infecções intestinais, o estímulo do sistema imune contra a invasão bacteriana, a prevenção e a melhoria dos distúrbios gastrintestinais; a redução de lipídios séricos e a prevenção do desenvolvimento de obesidade e câncer ${ }^{(5)}$.

Diversos fatores, incluindo a idade e o tipo de alimentação, sustentam a hipótese de que a presença da flora bífidodominante é benéfica não só para recém-nascidos e lactentes não-amamentados ao seio, mas também para crianças de outras faixas etárias. A oferta de alimentos com essa flora poderia ser concretizada por meio de duas formas distintas: a primeira é a oferta das próprias bactérias vivas, os chamados probióticos. Com esses agentes, uma vez interrompido o seu uso, há diminuição da flora protetora no trato intestinal. A segunda opção é a oferta do prebiótico, isso é, de substâncias que estimulam o crescimento das bactérias com ação benéfica na microflora do cólon, o que parece ser mais adequado ${ }^{(5)}$. $\mathrm{O}$ uso de ambos os nutrientes foi associado com redução na incidência e gravidade da diarreia infantil, particularmente nas gastrenterites virais e nas diarreias induzidas por antibióticos e consequentes às alergias alimentares ${ }^{(6)}$.

Dessa forma, como tentativa de manter uma flora bifidodominante no sistema digestivo de crianças desmamadas precocemente, os oligossacarídeos não-derivados do leite materno ganharam muita atenção nos últimos anos. Entretanto, como resultado da sua complexidade, os oligossacarídeos com estruturas idênticas aos oligossacarídeos humanos não estão ainda disponíveis como ingredientes dietéticos ${ }^{(1)}$. Assim, na busca atual por alternativas, as fórmulas lácteas e produtos para crianças têm sido suplementados com prebióticos $^{(7)}$.

As principais fontes de inulina e oligofrutose empregadas na indústria de alimentos são a chicória (Cichorium intybus) e a alcachofra de Jerusalém (Helianthus tuberosus) ${ }^{(8) . ~ A s s i m ~}$ como outras fibras da dieta, prebióticos como a inulina e a oligofrutose são resistentes à digestão na parte superior do trato intestinal, sendo posteriormente fermentados no cólon. Eles aumentam de volume como consequência do aumento da biomassa microbiana, que resulta de sua fermentação, e promovem aumento da frequência das evacuações, efeitos estes que confirmam a sua classificação no conceito atual de fibras da dieta. Quando adicionados como ingredientes funcionais a produtos alimentícios normais, os prebióticos típicos, como a inulina e a oligofrutose, exercem um papel primordial na fisiologia gastrintestinal ${ }^{(7)}$, reduzindo o risco de câncer de cólon, regulando a composição da microbiota intestinal e modulando as funções fisiológicas chaves, como a absorção de cálcio e o metabolismo lipídico ${ }^{(9)}$. A modulação promovida pelos prebióticos na microbiota intestinal é consequente a uma fermentação específica que altera sua composição, resultando em proliferação bacteriana com predomínio de bifidobactérias ${ }^{(8)}$.

Os prebióticos são digeridos parcialmente no intestino delgado, onde fortalecem o sistema imunológico ${ }^{(10)}$, inibem a multiplicação de patógenos e estimulam a proliferação e 
atividade de populações de bactérias desejáveis no cólon ${ }^{(11)}$, afetando beneficamente o hospedeiro com o desenvolvimento da flora bifidogênica ${ }^{(4)}$. Esses componentes atuam mais frequentemente no intestino grosso, embora possam ter também algum impacto sobre os microrganismos do intestino delgado ${ }^{(12)}$. Consequentemente, os prebióticos são considerados uma fonte natural de energia para o crescimento da flora bacteriana saudável do intestino ${ }^{(13)}$.

A modulação dietética da flora intestinal é hoje um dos tópicos principais de interesse nas ciências nutricionais. Executar essa modulação no período neonatal ou adiantado da infância, quando a programação imunológica ocorre, é um conceito relativamente novo. Os fruto-oligossacarídeos (FOS) e os galacto-oligossacarídeos (GOS) são os prebióticos cuja atividade bifidogênica já foi comprovada em adultos. Quando se trata de crianças, essa atividade tem sido pouco testada. Os prebióticos têm sido combinados em fórmulas lácteas infantis para reproduzir o efeito prebiótico dos oligossacarídeos humanos do leite materno, porém sua ação não está completamente esclarecida ${ }^{(14)}$.

Conceitualmente, a composição das fórmulas lácteas pretende se aproximar tanto quanto possível ao leite materno. Entretanto, há uma variedade de substâncias no leite humano - como aminoácidos, ácidos graxos, polipeptídios, nucleotídeos, oligossacarídeos, proteínas funcionais, hormônios, vitaminas e minerais - que são nutrientes de difícil incorporação nas fórmulas lácteas. Nesse sentido, propõe-se o desenvolvimento de uma suplementação orientada de prebióticos (com uma dosagem adequada, utilizada por um período correto) para as fórmulas lácteas infantis ${ }^{(15)}$.

Estudos indicam a possibilidade da suplementação segura de fórmulas infantis com FOS e GOS, no intuito de facilitar o trânsito intestinal de lactantes no primeiro e segundo semestres de vida ${ }^{(13)}$. Investigações preliminares indicam que os prebióticos podem ser adjuntos dietéticos úteis para controlar infecções no sistema gastrintestinal e podem ser utilizados para modificar uma composição microbiológica complexa, principalmente quando grupos de lactentes que usam fórmula láctea com adição de prebióticos são comparados a lactentes que recebem fórmula láctea sem prebióticos ${ }^{(16)}$.

Nesse sentido, a mistura de GOS neutros e de FOS de cadeia longa tem sido identificada como ingrediente prebiótico eficaz. Entretanto, o real papel dos prebióticos na infância e a dimensão de seus efeitos não estão totalmente esclarecidos, suscitando a realização de novas pesquisas com o intuito de melhorar a compreensão de seus efeitos a longo prazo na flora intestinal das crianças ${ }^{(17)}$.

A suplementação de prebióticos em fórmulas infantis ou de forma isolada tem aumentado significativamente nos últimos anos, impulsionando a indústria a destacar esses nutrientes nos rótulos dos alimentos e a difundir recomendações junto aos profissionais de saúde para utilização rotineira de seus produtos.

Em busca do conhecimento embasado sob a luz da evidência científica, o objetivo deste estudo é identificar e quantificar os reais efeitos da utilização de prebióticos (oligossacarídeos) na saúde da criança, salientando sua aplicação prática e os possíveis mecanismos envolvidos.

\section{Fontes de dados}

Este estudo é uma revisão da literatura sobre os efeitos da utilização de prebióticos na infância. Para obter as informações, artigos foram selecionados na base de dados virtuais de saúde da Biblioteca Regional de Medicina (Bireme), que incluem Lilacs, Medline e SciELO.

$\mathrm{Na}$ primeira etapa da busca científica foi utilizado o descritor de saúde 'oligossacarídeos'. O número de artigos encontrados indexados por esta palavra-chave foi de 16.612. De forma sequencial, foram selecionados os artigos que se referiam à pesquisa em humanos, os que estudaram a faixa etária de zero a 12 anos e os publicados no período entre 1998 e 2008, resultando, respectivamente em 5.803, 426 e 167 artigos.

Na segunda etapa, os 167 estudos escolhidos foram avaliados individualmente e os que tratavam do tema proposto foram selecionados, resultando em 29 estudos, que compuseram os resultados desta pesquisa. Em adição, 9 artigos não-evidenciados na primeira etapa foram identificados entre as referências dos 29 estudos selecionados. Além disso, foram adicionados 2 estudos apresentados em anais de congressos, ainda não publicados, consubstanciando o total de 40 estudos analisados e discutidos neste trabalho.

\section{Síntese dos dados}

Os dados encontrados nos 40 estudos selecionados para compor os resultados desta revisão, que abordam os efeitos da utilização de oligossacarídeos na saúde da criança, são apresentados e descritos na Tabela 1. Os efeitos encontrados foram compilados quantitativa e qualitativamente e podem ser verificados de forma resumida na Tabela 2 . 


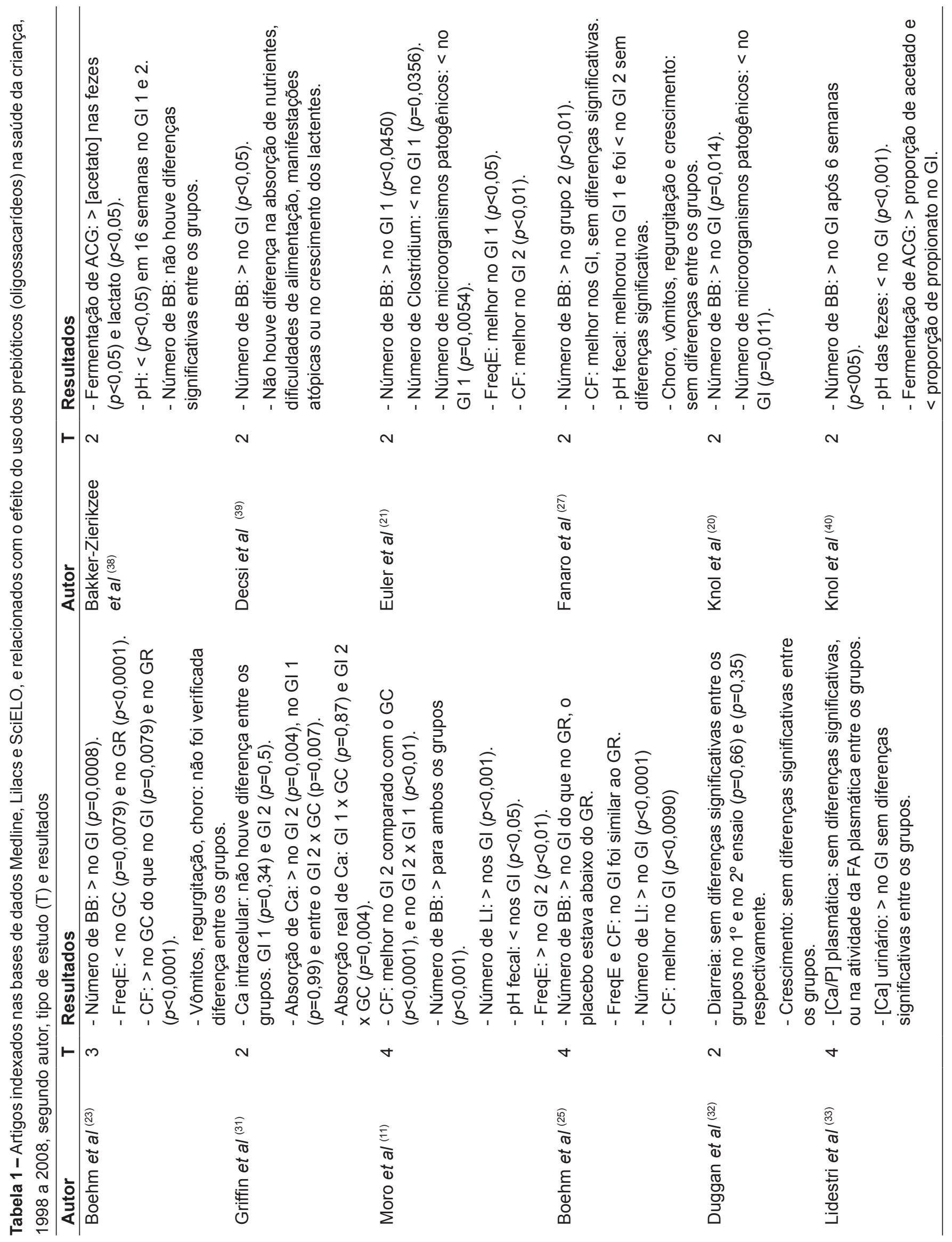




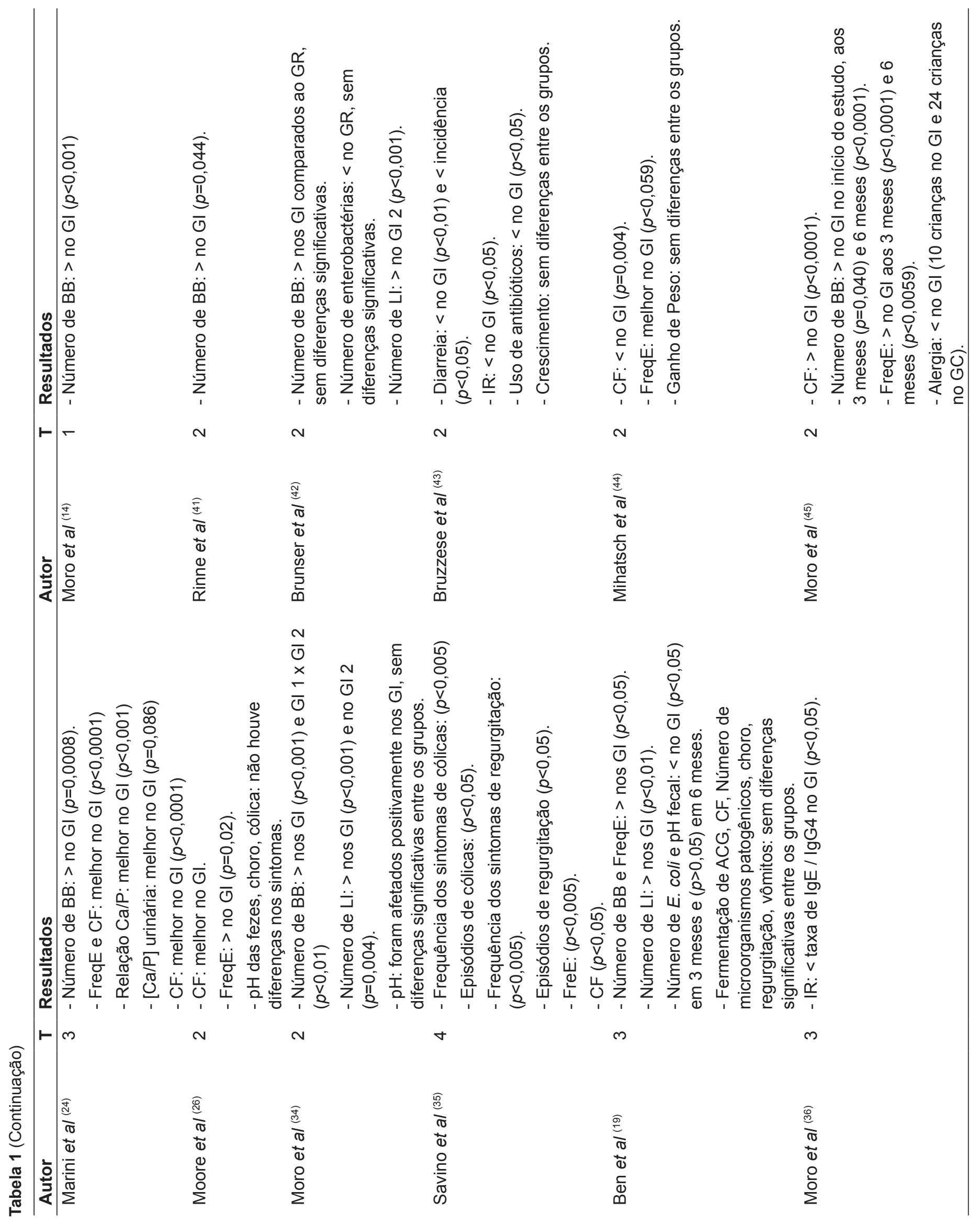




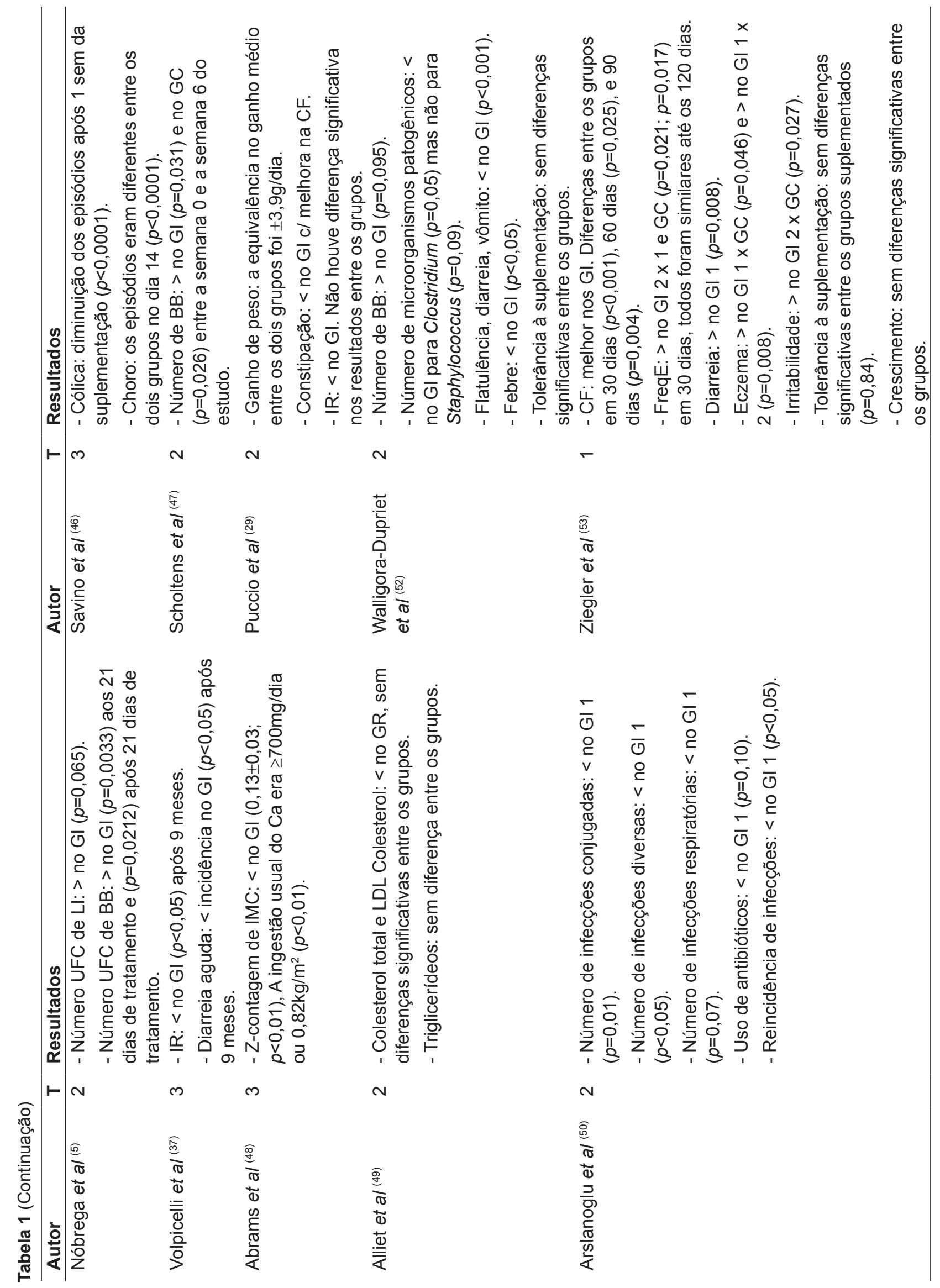




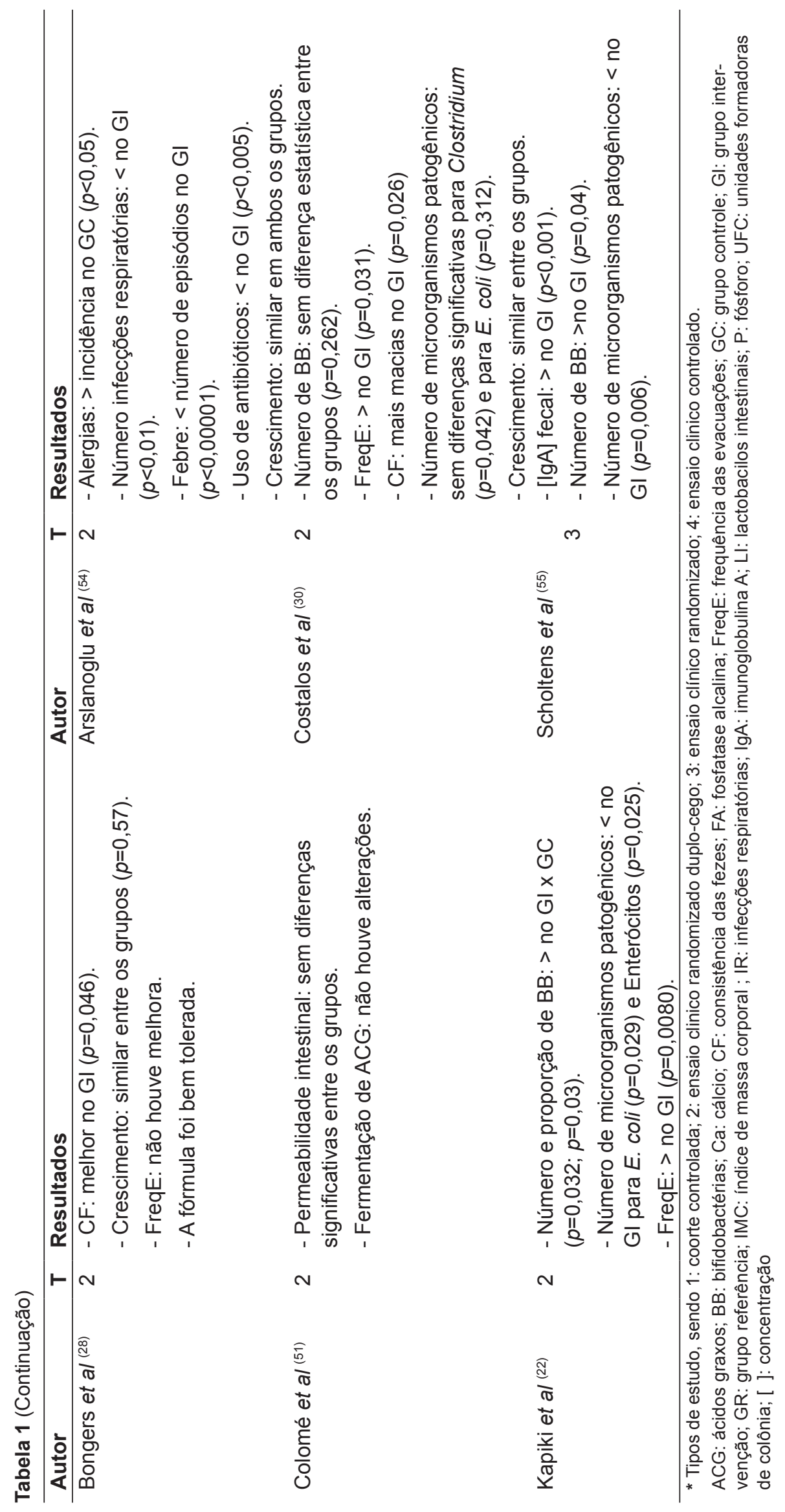


Tabela 2 - Efeitos clínicos dos prebióticos, segundo o número de estudos que o avaliaram e o efeito encontrado, dentre os 40 estudos analisados

\begin{tabular}{lcrrrrrr}
\hline \multirow{2}{*}{ Característica clínica } & $\mathbf{N}^{\circ}$ de estudos que & \multicolumn{2}{c}{ Efeito positivo } & \multicolumn{2}{c}{ Efeito negativo } & \multicolumn{2}{c}{ Efeito nulo } \\
\cline { 2 - 8 } & avaliaram o efeito & $\mathbf{n}$ & $\%$ & $\mathbf{n}$ & $\%$ & $\mathbf{n}$ & $\%$ \\
\hline Absorção de cálcio & 5 & 3 & 75 & 0 & 0 & 2 & 25 \\
Alergias & 4 & 3 & 75 & 0 & 0 & 1 & 25 \\
Cólica/choro & 8 & 4 & 50 & 0 & 0 & 4 & 50 \\
Consistência das fezes & 13 & 13 & 100 & 0 & 0 & 0 & 0 \\
Contagem de lipídeos séricos & 1 & 0 & 0 & 0 & 0 & 1 & 100 \\
Crescimento/ganho de peso & 13 & 0 & 0 & 1 & 8 & 12 & 92 \\
Diarreia & 5 & 4 & 80 & 0 & 0 & 1 & 20 \\
Febre & 2 & 2 & 100 & 0 & 0 & 0 & 0 \\
Fermentação de ácidos graxos & 4 & 3 & 75 & 0 & 0 & 1 & 25 \\
Flatulência & 1 & 1 & 100 & 0 & 0 & 0 & 0 \\
Frequência das evacuações & 12 & 10 & 83 & 0 & 0 & 2 & 17 \\
Infecções diversas & 1 & 1 & 100 & 0 & 0 & 0 & 0 \\
Infecções respiratórias & 6 & 6 & 100 & 0 & 0 & 0 & 0 \\
Irritabilidade & 1 & 1 & 100 & 0 & 0 & 0 & 0 \\
No bifidobactérias & 23 & 21 & 91 & 0 & 0 & 2 & 9 \\
No microrganismos patogênicos & 10 & 7 & 70 & 0 & 0 & 3 & 30 \\
Permeabilidade intestinal & 1 & 0 & 0 & 0 & 0 & 1 & 100 \\
pH das fezes & 6 & 5 & 83 & 0 & 0 & 1 & 17 \\
Regurgitação & 4 & 1 & 25 & 0 & 0 & 3 & 75 \\
Tolerância à suplementação & 9 & 9 & 100 & 0 & 0 & 0 & 0 \\
Uso de antibióticos & 5 & 4 & 80 & 0 & 0 & 1 & 20 \\
Vômitos & 4 & 1 & 25 & 0 & 0 & 3 & 75 \\
\hline
\end{tabular}

\section{Discussão}

Ao adicionar prebióticos às suas formulações, o objetivo da indústria de alimentos é tentar fazê-las parecer o máximo possível com o leite humano, de modo a atender à demanda da utilização em crianças que não recebem ou que recebem parcialmente esse alimento, fundamental para o desenvolvimento nos primeiros anos de vida. Dessa forma, modifica-se a flora intestinal e seus efeitos bacteriológicos, reproduzindo os benefícios funcionais do leite humano.

Os prebióticos podem ser usados a partir do nascimento. Entretanto, são poucos os dados publicados sobre a avaliação de sua utilização em produtos alimentares para crianças. $\mathrm{O}$ comitê da European Society for Paediatric Gastroenterology, Hepatology and Nutrition (ESPGHAN) não autoriza recomendações quanto ao uso de oligossacarídeos na infância para finalidades preventivas ou terapêuticas ${ }^{(18)}$.

Segundo dados obtidos na presente revisão, durante sua administração em produtos alimentares, os oligossacarídeos prebióticos apresentaram características benéficas, que influenciam no aumento do número total de bifidobactérias ${ }^{(19,20)}$, redução do número de microorganismos patogênicos no intestino ${ }^{(21,22)}$, melhora da consistência das fezes ${ }^{(23,24)}$ e frequência das evacuações. Dessa forma, a presença desses efeitos benéficos, observados em curto prazo em crianças constipadas, sugere que alguma recomendação poderia ser feita nesse sentido na prática clínica.

Quase a totalidade dos estudos (91\%), que quantificaram as bifidobactérias nas fezes, verificaram aumento significativo do número desses componentes benéficos, sendo que, em alguns casos, o número foi superior ao encontrado em crianças saudáveis amamentadas. De forma similar, sete de dez pesquisas (70\%) identificaram redução do número de microorganismos patogênicos na flora intestinal das crianças suplementadas com oligossacarídeos. Esses resultados sugerem fortemente que a suplementação com prebióticos é eficaz para diminuir o número de microorganismos patogênicos e aumentar o número de bifidobactérias nas fezes, promovendo adequação da flora intestinal e melhora na dinâmica do sistema digestivo.

Quanto à consistência das fezes, foram encontrados efeitos benéficos nos $13(100 \%)$ estudos que analisaram o tema. Os 
resultados foram verificados em faixas etárias distintas, com a presença de fezes mais macias, o que proporcionou a melhora e prevenção dos quadros de constipação, contribuindo para o bem-estar das crianças estudadas. Paralelamente, dez (83\%) de 12 estudos realizados em lactentes identificaram melhora significativa na frequência das evacuações. Tais achados sugerem que a suplementação com prebióticos pode interferir positivamente nas características das fezes e no equilíbrio da frequência das evacuações.

Foi verificado um menor número de casos de alergias em três de quatro estudos, incluindo melhora na incidência de eczema atópico - porém a faixa etária estudada foi variável, dificultando a extrapolação dos resultados encontrados. Infecções diversas foram avaliadas em apenas um estudo, que verificou melhora; infecções respiratórias com melhora em seis $(100 \%)$ dos estudos realizados, indicando que a suplementação com prebióticos parece reforçar direta ou indiretamente o sistema imunológico dos lactentes. Apesar dos resultados apresentados apontarem para efeitos de tendência benéfica quanto a esses quadros mórbidos, a execução de mais estudos parece necessária para avaliar a real dimensão desses benefícios, uma vez que a utilização da suplementação possivelmente auxilia o controle de doenças e poucos estudos foram realizados com esse fim até o momento.

Com relação aos demais efeitos, como febre, os dois estudos que pesquisaram o tema verificaram melhora no número de episódios de febre em crianças, associada à redução do número de infecções; quanto à irritabilidade e flatulência, apenas um estudo investigou e encontrou melhora; sobre a fermentação de ácidos graxos, três dos quatro estudos que verificaram esse efeito notaram melhora na fermentação de ácidos graxos nas fezes de forma similar às concentrações de crianças em aleitamento materno; no caso da diarreia, quatro dos cinco estudos realizados verificaram menor incidência e prevalência de episódios de diarreia, um dos estudos não encontrou efeito relacionado em crianças que ainda eram amamentadas e que foram suplementadas com cereal contendo prebióticos, o que sugere que crianças amamentadas não se beneficiam com a suplementação; com relação ao $\mathrm{pH}$ das fezes, quatro dos cinco estudos que avaliaram o tema observaram $\mathrm{pH}$ fecal diminuído em crianças; sobre a utilização de antibióticos, quatro dos cinco estudos que analisaram este item encontraram redução do uso destes medicamentos; quanto à regurgitação, em apenas um dos quatro estudos que avaliaram esse efeito foi constatada a redução na frequência dos sintomas e no número de episódios; com relação aos vômitos, somente um em quatro estudos verificou melhora na prevalência deste sintoma; sobre a permeabilidade intestinal, apenas um estudo avaliou esse efeito e verificou pequeno aumento da permeabilidade intestinal, sem alterações significativas, considerando nulo o seu efeito; à respeito da cólica e choro, quatro em oito trabalhos que estudaram esses eventos verificaram a redução de seus episódios; finalmente, quanto à contagem de lipídeos séricos, apenas um estudo avaliou este efeito e não verificou nenhuma diferença no colesterol total e no LDL colesterol. O pequeno número de estudos que investigaram tais efeitos não permite uma conclusão definitiva quanto ao benefício da suplementação com prebióticos. Assim, esses resultados apontam para a necessidade de novas investigações com este intuito.

Alguns estudos verificaram melhora na absorção do cálcio. Entretanto, os resultados são contraditórios, sendo que apenas dois entre quatro estudos encontraram efeitos positivos. Um dos ensaios resultou em menor absorção de cálcio com a suplementação, porém associada ao baixo consumo - o que não possibilita averiguar se o suplemento interferiu nesse processo. Faz-se necessária a realização de estudos adicionais para verificar se a suplementação promove impacto no crescimento em longo prazo.

Ressalta-se que grande parte dos estudos avaliados não analisou a absorção de outras substâncias, entre nutrientes, minerais e oligoelementos (exceto cálcio), concomitante à ingestão dos oligossacarídeos prebióticos. A ausência desse controle pode ter contribuído para uma interpretação imprecisa dos efeitos estudados, uma vez que, na dinâmica digestiva, há interação constante entre os nutrientes existentes na luz intestinal.

Doze (92\%) dos 13 estudos que investigaram o ganho ponderal e o crescimento das crianças não constataram alterações com a utilização da suplementação. Entretanto, um deles verificou deficiência de crescimento em pré-púberes, mesmo após o término da suplementação. Tal situação indica a necessidade de novas investigações para verificar os possíveis efeitos em diversas idades em longo prazo.

Poucos estudos avaliaram a tolerância à suplementação em crianças. A faixa etária pesquisada e as doses administradas são muito variadas, sendo que há a indicação apenas da ausência de desconforto mediante a administração da suplementação. Dentre as quantidades toleradas e com respostas positivas à suplementação encontramos: $10 \mathrm{~g} / \mathrm{L}$ durante 28 dias em prematuros em nutrição enteral ${ }^{(23,25)} ; 0,74$ e $3 \mathrm{~g}$ durante 28 dias em lactentes amamentados com idade média de $32,5 \operatorname{semanas}^{(26)}$; 1,5 e $3 \mathrm{~g}$ durante sete dias em lactentes com idade entre 2 a 6 semanas $^{(21)} ; 0,2$ e $0,6 \mathrm{~g} / 100 \mathrm{~mL}$ em lactentes saudáveis ${ }^{(27)}$; 
$0,8 \mathrm{~g} / 100 \mathrm{~mL}$ durante três semanas em lactentes constipados com idade entre 3-20 semanas ${ }^{(28)} ; 0,4 \mathrm{~g} / \mathrm{dL}$ durante 14 dias em prematuros saudáveis $^{(22)} ; 4 \mathrm{~g} / \mathrm{L}$ durante 112 dias em lactentes saudáveis com idade inferior a 4 meses ${ }^{(29)} ; 0,4 \mathrm{~g} / 100 \mathrm{~mL}$ durante 14 dias em neonatos saudáveis com até 14 dias de vida ${ }^{(30)}$. Com base nestes dados, percebe-se a necessidade de elaborar e executar pesquisas que verifiquem essa tolerância em diferentes faixas etárias, em quantidades variadas do suplemento e considerando as características clínicas de saúde em que as crianças se encontram. Sem isso, não parece possível inferir definitivamente qual seria a dose segura de prebióticos a ser administrada às crianças suplementadas.

Cabe ressaltar que a metodologia utilizada para a execução do presente estudo evidenciou pesquisas cujos achados foram publicados na última década, o que pode ter limitado a busca dos objetivos propostos. Entretanto, os resultados aqui apresentados parecem representar o estado da arte dos efeitos do uso de prebióticos na saúde da criança, uma vez que esse tema é relativamente recente na literatura científica e na prática assistencial.

Com base nos dados verificados nesta revisão, e apesar de os prebióticos oferecerem efeitos benéficos em determinadas situações, conclui-se que alguns aspectos duvidosos devem ser mais bem investigados de forma a avaliar a real eficácia e segurança da utilização desse suplemento em caráter imediato e a longo prazo.

Os profissionais da área da saúde devem constantemente buscar informações, com evidências científicas dos efeitos obtidos com a utilização de prebióticos para efetuar a prescrição desses suplementos. Nesse contexto, o comportamento crítico parece importante, pois apesar da existência de efeitos promissores, alguns carecem de estudos definitivos, o que deve impulsionar profissionais a realizar mais pesquisas de forma a investigar tais aspectos. Além disso, aconselha-se verificar a relação custo-benefício na prescrição dessa suplementação em crianças, uma vez que se promove uma aquisição adiantada das bifidobactérias remanescentes, que é questionável em crianças pré-termo.

Em adição, a indústria de alimentos deve ter cautela na incorporação de oligossacarídeos prebióticos em produtos alimentares ou em fórmulas lácteas para crianças, uma vez que efeitos provenientes da sua utilização em longo prazo ainda não estão esclarecidos.

\section{Referências bibliográficas}

1. Boehm G, Stahl B. Oligosaccharides from milk. J Nutr 2007;137:847S-9S.

2. Morrow AL, Ruiz-Palacios GM, Jiang X, Newburg DS. Human-milk glycans that inhibit pathogen binding protect breast-feeding infants against infectious diarrhea. J Nutr 2005;135:1304-7.

3. Gudiel-Urbano M, Goni I. Oligosacaridos de la leche humana: papel en la salud y en el desarrollo del lactante. Arch Latinoam Nutr 2001;51:332-9.

4. Coppa GV, Bruni S, Morelli L, Soldi S, Gabrielli O. The first prebiotics in humans: human milk oligosaccharides. J Clin Gastroenterol 2004;38:S80-3.

5. Nóbrega FJ, Trabulsi LR, Keller R, Franzolin MR, Alves RC, Santos MF et al. Efeitos do prebiótico (oligossacarídeo) em leite em pó modificado na flora intestinal: comparação com leite em pó modificado sem prebiótico em estudo duplo-cego. Rev Paul Pediatr 2004;22:205-11.

6. Chen CC, Walker WA. Probiotics and prebiotics: role in clinical disease states. Adv Pediatr 2005;52:77-113.

7. Roberfroid M. Functional food concept and its application to prebiotics. Dig Liver Dis 2002;34:S105-10.

8. Kaur N, Gupta AK. Applications of inulin and oligofructose in health and nutrition. J Biosci 2002;27:703-14.

9. Vandenplas Y. Oligosaccharides in infant formula. Br J Nutr 2002;87: S293-6.

10. Boehm G, Jelinek J, Stahl B, van Laere K, Knol J, Fanaro S et al. Prebiotics in infant formulas. J Clin Gastroenterol 2004;38:S76-9.

11. Moro G, Minoli I, Mosca M, Fanaro S, Jelinek J, Stahl B et al. Dosage-related bifidogenic effects of galacto- and fructooligosaccharides in formula-fed term infants. J Pediatr Gastroenterol Nutr 2002;34:291-5.

12. Andryseca MV, Bragazzi S, Cassina CV, Di Gregorio S, Ursúa V. Fructooligosacáridos en el organismo. Diaeta (B Aires) 2005;23:25-40.

13. Agostoni C, Axelsoson I, Goulet O, Koletzko B, Michaelsen KF, Puntis JW et al. Prebiotic oligosaccharides in dietetic products for infants: a commentary by the ESPGHAN Committee on Nutrition. J Pediatr Gastroenterol Nutr 2004;39:465-73.

14. Moro GE, Stahl B, Fanaro S, Jelinek J, Boehm G, Coppa GV. Dietary prebiotic oligosaccharides are detectable in the faeces of formula-fed infants. Acta Paediatr Suppl 2005;94:27-30.

15. Böhles H, Gebhardt B, Beeg T. Reflections about possible nutritional supplements in infant milk formula. Z Ernahrungswiss 1998;37:132-46.

16. Morais MB, Jacob CM. O papel dos probióticos e prebióticos na prática pediátrica. J Pediatr (Rio J) 2006;82:S189-97.

17. Salvini F, Granieri L, Gemmellaro L, Giovannini M. Probiotics, prebiotics and child health: where are we going? J Int Med Res 2004;32:97-108.

18. European Committee, Scientific Committee on Food [homepage on the internet]. Baby milk action comments on the SCF Report on the revision of essential requirements of infant formulae and follow-on formulae (SCF/CS/ NUT/IF/65) [cited 2008 Aug 10]. Available from: http://www.babymilkaction. org/pdfs/scf0503.pdf/.

19. Ben XM, Zhou XY, Zhao WH, Yu WL, Pan W, Zhang WL et al. Supplementation of milk formula with galacto-oligosaccharides improves intestinal micro-flora and fermentation in term infants. Chin Med J (Engl) 2004;117:927-31.

20. Knol J, Boehm G, Lidestri M, Negretti F, Jelinek J, Agosti M et al. Increase of faecal bifidobacteria due to dietary oligosaccharides induces a reduction of clinically relevant pathogen germs in the faeces of formula-fed preterm infants. Acta Paediatr 2005;94:31-3.

21. Euler AR, Mitchell DK, Kline R, Pickering LK. Prebiotic effect of fructooligosacchride supplemented term infant formula at two concentrations compared with unsupplemented formula and human milk. J Pediatr Gastroenterol Nutr 2005;40:157-64.

22. Kapiki A, Costalos C, Oikonomidou C, Triantafyllidou A, Loukatou E, Pertrohilou $\mathrm{V}$. The effect of a fructo-oligosaccharide supplemented formula on gut flora of 
preterm infants. Early Hum Dev 2007;83:335-9

23. Boehm G, Lidestri M, Casetta P, Jelinek J, Negretti F, Stahl B et al. Supplementation of a bovine milk formula with an oligosaccharide mixture increases counts of faecal bifidobacteria in preterm infants. Arch Dis Child Fetal Neonatal Ed 2002;86:F178-81.

24. Marini A, Negretti F, Boehm G, Li Destri M, Clerici-Bagozzi D, Mosca F et al. Pro- and pre-biotics administration in preterm infants: colonization and influence on faecal flora. Acta Paediatr Suppl 2003;91:80-1.

25. Boehm G, Fanaro S, Jelinek J, Stahl B, Marini A. Prebiotic concept for infant nutrition. Acta Paediatr Suppl 2003;91:64-7.

26. Moore N, Chao C, Yang LP, Storm H, Oliva-Hemker M, Saavedra JM. Effects of fructo-oligosaccharide-supplemented infant cereal: a double-blind, randomized trial. Br J Nutr 2003;90:581-7.

27. Fanaro S, Jelinek J, Stahl B, Boehm G, Kock R, Vigi V. Acidic oligosaccharides from pectin hydrolysate as new component for infant formulae: effect on intestinal flora, stool characteristics, and $\mathrm{pH}$. J Pediatr Gastroenterol Nutr 2005;41:186-90.

28. Bongers ME, Lorijn F, Reitsma JB, Groeneweg M, Taminiau JA, Benninga MA. The clinical effect of a new infant formula in term infants with constipation: a double-blind, randomized cross-over trial. Nutr J 2007;6:8.

29. Puccio G, Cajozzo C, Meli F, Rochat F, Grathwohl D, Steenhout P. Clinical evaluation of a new starter formula for infants containing live Bifidobacterium longum BL999 and prebiotics. Nutrition 2007;23:1-8.

30. Costalos C, Kapiki A, Apostolou M, Papathoma E. The effect of a prebiotic supplemented formula on growth and stool microbiology of term infants. Early Hum Dev 2008;84:45-9.

31. Griffin IJ, Davila PM, Abrams SA. Non-digestible oligosaccharides and calcium absorption in girls with adequate calcium intakes. Br J Nutr 2002;87: S187-91.

32. Duggan C, Penny ME, Hibberd P, Gil A, Huapaya A, Cooper A et al. Oligofrutose-supplemented infant cereal: 2 randomized, blinded, communitybased trials in Peruvian infants. Am J Clin Nutr 2003;77:937-42.

33. Lidestri M, Agosti M, Marini A, Boehm G. Oligosaccharides might stimulate calcium absorption in formula-fed preterm infants. Acta Paediatr Suppl 2003;91:91-2.

34. Moro GE, Mosca F, Miniello V, Fanaro S, Jelinek J, Stahl B et al. Effects of a new mixture of prebiotics on faecal flora and stools in term infants. Acta Paediatr Suppl 2003;91:77-9.

35. Savino F, Cresi F, Maccario S, Cavallo F, Dalmasso P, Fanaro S et al. "Minor" feeding problems during the first months of life: effect of a partially hydrolysed milk formula containing fructo-and galacto-oligosaccharides. Acta Paediatr Suppl 2003;92:86-90.

36. Moro G, Garssen J, M'Rabet L, Faber J, Stahl B et al. Role of prebiotics in the immune modulation: clinical results. Proceedings of the 21 st International Symposium on Neonatal Intensive Care; 2004 Nov; Milan, Italy. p. 67-77.

37. Volpicelli M, Bruzzese E, Gaudiello G, Salvini F, Bisceglia M et al. Efficiency of a prebiotic formula in the prevention of intestinal and extra intestinal infections during the first year of life (Italian translation). Proceedings of the 5th National Congress of the Italian Society of Paediatric Infectious Diseases; 2005 Nov; Naples, Italy.

38. Bakker-Zierikzee AM, Alles MS, Knol J, Kok FJ, Tolboom JJ, Bindels JG. Effects of infant formula containing a mixture of galacto-and fructo-oligosaccharides or viable Bifidobacterium animalis on the intestinal microflora during the first 4 months of life. Br J Nutr 2005;94:783-90.

39. Decsi T, Arató A, Balogh M, Dolinay T, Kanjo AH, Szabó E et al. Randomised placebo controlled double blind study on the effect of prebiotic oligosaccharides on intestinal flora in healthy infants. Orv Hetil 2005;146:2445-50.

40. Knol J, Scholtens P, Kafka C, Steenbakkers J, Gro S, Helm K et al. Colon microflora in infants fed formula with galacto- and fructo-oligosaccharides: more like breast-fed infants. J Pediatr Gastroenterol Nutr 2005;40:36-42.

41. Rinne MM, Gueimonde M, Kalliomaki M, Hoppu U, Salminen SJ, Isolauri E. Similar bifidogenic effects of prebiotic-supplemented partially hydrolyzed infant formula and breastfeeding on infant gut microbiota. FEMS Immunol Med Microbiol 2005;43:59-65.

42. Brunser O, Figueroa G, Gotteland M, Haschke-Becher E, Magliola C, Rochat $\mathrm{F}$ et al. Effects of probiotic or prebiotic suplemented milk formulas on fecal microbiota composition of infants. Asia Pac J Clin Nutr 2006;15:368-76.

43. Bruzzese E, Volpicelli M, Salvini F, Bisceglias M, Lionetti P, Cinquetti M et al. Early administration of GOS/FOS prevents intestinal and respiratory infections in infants. J Pediatr Gastroenterol Nutr 2006;42:E95.

44. Mihatsch WA, Hoegel J, Pohlandt F. Prebiotic oligosaccharides reduce stool viscosity and accelerate gastrointestinal transport in preterm infants. Acta Paediatr 2007;95:843-8.

45. Moro G, Arslanoglu S, Stahl B, Jelinek J, Wahn U, Boehm G. A mixture of prebiotic oligosaccharides reduces the incidence of atopic dermatitis during the first six months of age. Arch Dis Child 2006;91:814-9.

46. Savino F, Palumeri E, Castagno E, Cresi F, Dalmasso P, Cavallo F et al. Reduction of crying episodes owing to infantile colic: a randomized controlled study on the efficacy of a new infant formula. Eur J Clin Nutr 2006;60: 1304-10.

47. Scholtens PA, Alles MS, Bindels JG, van der Linde EG, Toolbom JJ, Knol J. Bifidogenic effects of solid weaning foods with added prebiotic oligosaccharides: a randomised controlled clinical trial. J Pediatr Gastroenterol Nutr 2006;42:553-9.

48. Abrams SA, Griffin IJ, Hawthorne KM, Ellis KJ. Effect of prebiotic supplementation and calcium intake on body mass index. J Pediatr 2007;151:293-8.

49. Alliet $\mathrm{P}$, Scholtens $\mathrm{P}$, Raes $\mathrm{M}$, Hensen $\mathrm{K}$, Jongen $\mathrm{H}$, Rummens JL et al. Effect of prebiotic galacto-oligosaccharide, long-chain fructo-oligosaccharide infant formula on serum cholesterol and triacylglycerol levels. Nutrition 2007;23: 719-23.

50. Arslanoglu S, Moro GE, Boehm G. Early supplementation of prebiotic oligosaccharides protects formula-fed infants against infections during the first 6 months of life. J Nutr 2007;137:2420-4.

51. Colomé G, Sierra C, Blasco J, García MV, Valverde E, Sánchez E. Intestinal permeability in different feedings in infancy. Acta Paediatr 2007;96:69-72.

52. Waligora-Dupriet AJ, Campeotto F, Nicolis I, Bonet A, Soulaines P, Dupont C et al. Effect of oligofructose supplementation on gut microflora and well-being in young children attending a day care centre. Int J Food Microbiol 2007;113: 108-13.

53. Ziegler E, Vanderhoof JA, Petschow B, Mitmesser SH, Stolz SI, Harris CL et al. Term infants fed formula supplemented with selected blends of prebiotics grow normally and have soft stools similar to those reported for breast-fed infants. J Pediatr Gastroenterol Nutr 2007;44:359-64.

54. Arslanoglu S, Moro GE, Schmitt J, Tandoi L, Rizzardi S, Boehm G. Early dietary intervention with a mixture of prebiotic oligosaccharides reduces the incidence of allergic manifestations and infections during the first two years of life. J Nutr 2008;138:1091-5.

55. Scholtens PA, Alliet P, Raes M, Alles MS, Kroes H, Boehm G et al. Fecal secretory immunoglobulin $A$ is increased in healthy infants who receive a formula with short-chain galacto-oligosaccharides and long-chain fructooligosaccharides. J Nutr 2008;138:1141-7. 